\title{
Synchronous double primary cancer of the lung and nasal vestibule: A case report and literature review
}

\author{
HUAI JUN JI $^{1}$, QI ZANG ${ }^{2}$, WEI WANG ${ }^{2}$, NING BO SUN ${ }^{1}$, MING CAO $^{2}$, QIANG ZHU ${ }^{2}$ and ZHONG MIN JIANG ${ }^{2}$ \\ ${ }^{1}$ Division of Surgery, Graduate Department, Weifang Medical College, Weifang, Shandong 261031; \\ ${ }^{2}$ Department of Thoracic Surgery, Qianfoshan Hospital Affiliated to Shandong University, Jinan, Shandong 250014, P.R. China
}

Received August 19, 2015; Accepted February 22, 2016

DOI: $10.3892 / \operatorname{mco} .2016 .799$

\begin{abstract}
Squamous cell carcinoma (SCC) of the nasal vestibule is a rare tumor entity, and its occurrence combined with lung cancer is even rarer. Thus, several patients are often initially misdiagnosed or remain undiagnosed. This is the case report of a 55-year-old male patient who presented to our hospital with a neoplasm in the left lung. The patient was treated with left upper pulmonary lobectomy and the subsequent histopathological examination of the surgical specimen revealed a poorly differentiated SCC. On postoperative week 4, the patient presented with purulent and bloody discharge from the left nostril and was misdiagnosed with an upper jaw cyst. After another 3 weeks, the patient was re-admitted to the hospital with a mass of left nostril and nasal congestion. Tru-Cut biopsies from the nasal area and histopathological examination revealed a moderately differentiated SCC. According to the clinical presentation and the histopathological findings, the patient was diagnosed with double primary cancer of the lung and the nasal vestibule. The mass of the left nostril was significantly reduced in size with radiotherapy. To the best of our knowledge, there is no similar case previously reported in the literature. Due to the rarity of $\mathrm{SCC}$ of the nasal vestibule concomittant with lung cancer, we herein present this case report with a review of the relevant literature and investigation of the clinical characteristics.
\end{abstract}

\section{Introduction}

Multiple primary cancers are defined as $\geq 2$ separate original cancers, simultaneously or successively occurring in the same or different organs. Double primary cancer is the most common type of multiple primary cancer and it is classified as synchronous carcinoma (SC) and metachronous

Correspondence to: Professor Zhong Min Jiang, Department of Thoracic Surgery, Qianfoshan Hospital Affiliated to Shandong University, No. 16766 Jingshi Road, Jinan, Shandong 250014, P.R. China

E-mail: qyjzm@sina.cn

Key words: double primary cancer, squamous cell carcinoma, lung cancer, nasal vestibule, radiotherapy, misdiagnosis carcinoma (MC). SC is used to describe the second primary tumor when it is diagnosed simultaneously or within 6 months of the diagnosis of the first primary tumor. $\mathrm{MC}$ is is used to describe the second cancer when it is diagnosed $>6$ months after the first primary cancer (1). The first double primary cancer was reported in 1889 by Billroth (2), whose standard of the diagnosis was that each tumor should have a different localization and independent histological appearance. In 1932, Warren and Gates established new criteria, which are still applied today: i) Both cancers must be definitively malignant according to the histopathological examination; ii) the cancers must be histologically different; and iii) it must be established that the second cancer is not a metastatic lesion of the first (3). The incidence of multiple primary cancers appears to have increased significantly. Approximately $10 \%$ of cancer patients develop another primary cancer within 10 years of the first surgery (4). However, double primary cancer of the lung and nasal vestibule is uncommon. We herein describe an extremely rare case of synchronous double primary cancer involving the left nasal vestibule and the left lung, with a review of the literature.

\section{Case report}

A 55-year-old man presented to the Qianfoshan Hospital on April 8, 2015, where he was diagnosed with a neoplasm of the left lung. The patient had noticed bloodstained sputum 5 years prior, which later disappeared. However, the symptom recurred 2 weeks prior to admission, with accompanying intermittent pain in the left hemithorax. The patient also reported loss of appetite and weight loss of $3 \mathrm{~kg}$ over the last 2 months. The patient had been a chronic smoker (40 cigarettes/day) and consumed alcohol (500 gr/day) for 20 years. On physical examination, there was no peripheral lymph node enlargement. The laboratory tests revealed a squamous cell carcinoma (SCC) antigen level of $2.1 \mathrm{ng} / \mathrm{ml}$ (normal value: $<1.5 \mathrm{ng} / \mathrm{ml}$ ); the other blood tests were all within the normal range. Contrast-enhanced computed tomography (CECT) examination of the thorax revealed a mass sized $\sim 5.3 \times 3.7 \mathrm{~cm}$ in the upper lobe of the left lung (Fig. 1A). Other radiological examinations, including whole-body emission CT, did not reveal any evidence of distant metastasis, and a CT scan of the brain was unremarkable (Fig. 1B). The patient was treated with left upper lobectomy and mediastinal lymph 
node sampling. Following histopathological examination, the lesion was diagnosed as poorly differentiated SCC (pT3N0M0, IIb) (Fig. 1C). No lymph nodes were infiltrated. Three weeks later, the patient received one cycle of adjuvant chemotherapy with regular doses of nedaplatin (120 mg/day) and gemcitabine (1,600 mg/day).

On a regular visit after chemotherapy week 1 , the patient presented with a small amount of purulent and bloody discharge from the left nostril, which was first diagnosed as an upper jaw cyst, and was treated with cefathiamidine antibiotics for two days ( $2 \mathrm{~g}$, twice per day). Three weeks later, the patient was re-admitted with bloody discharge from the left nostril, a mass in the nose and nasal congestion. The CT examination revealed a mass over the left nasal vestibule (Fig. 1D). Tru-Cut biopsies from the nasal area were performed and histopathological examination revealed a moderately differentiated SCC (pT2N0M0) (Fig. 1E). According to the history, the patient was finally diagnosed with double primary cancer of the lung and nasal vestibule. Following systemic evaluation, external-beam radiation treatment was recommended and the nasal tumor was significantly reduced after one cycle of radiotherapy.

\section{Discussion}

Lung cancer is one of the most commonly diagnosed types of cancer affected by population aging and changes in lifestyle, such as smoking and physical inactivity (5). Non-small-cell lung cancer is the leading cause of cancer-related mortality worldwide (5). The number of new cases of lung cancer diagnosed annually worldwide is $\sim 1.5$ million (6). Surgery, radiotherapy, chemotherapy and immunotherapy are the four most widely employed cancer treatments (7). However, Han et al reported that the 5-year survival rate remains very poor (8). Nasal vestibular carcinomas are uncommon and are typically SCCs. They display a natural history similar to that of skin cancers and their prognosis is more favorable compared with other cancers of the nasal cavity (9). Nasal vestibular carcinomas are often located in the nasal floor, the nasal dome, the medial crus of the lower lateral cartilage and the inner lining of the alar wing (lateral crus), and account for $<3.8 \%$ of all nasal mucosal tumors and $1 \%$ of all malignant tumors of the head and neck (10-12). Human papillomavirus infection and smoking are considered to be risk factors (12). However, their prognosis remains better compared with that of other sinonasal malignant tumors, such as maxillary sinus and frontal sinus malignant tumor $(13,14)$. The 5 -year recurrence-free survival rates range widely between 20 and $92 \%(15-17)$.

To the best of our knowledge, cases of double primary cancer of the nasal vestibule and lung are extremely rare. Thus, a number of patients may be misdiagnosed or remain undiagnosed in the first visit, resulting in a delay in treatment. The patient in the present study was originally misdiagnosed with an upper jaw cyst. Taking into consideration the clinical symptoms, signs and the correlation of the examinations with the opinions of experts, including a thoracic surgeon, otolaryngologist and pathologist, the patient was finally diagnosed with double primary cancer of the lung and nasal vestibule: i) We were able to determine that the cancer of lung occurred earlier compared with that of the nasal vestibule and the possibility of pulmonary metastatic tumor was ruled out, as the patient had first presented with bloodstained sputum 5 years prior and the CECT examination of the thorax revealed a large mass, whereas the CT scan of the brain was unremarkable. ii) Head and neck metastasis from lung cancer is an unusual event, particularly to the nasal vestibule. The larynx is a terminally located organ in regard to vascular and lymphatic circulation (18). Primary pulmonary cancer metastasis to the larynx is very rare through the vascular or lymphatic route, and metastasis to the nasal vestibule is even rarer. In addition, on preoperative clinical examination there was no cervical lymph node enlargement and the test results revealed no evidence of distant metastasis. iii) Although the histopathological diagnosis of both tumors was SCC, the degree of pathological differentiation (the level of malignancy) was different. Thus, metastatic carcinoma was deemed unlikely.

However, identification of the primary or metastatic nature of the nasal vestibular SCC is difficult. As there is no definitive evidence, it may be argued that a history of malignancy does not necessarily suggest that a nasal vestibular lesion is primary in nature. It is well-known among pathologists that there are currently no immunohistochemical markers for the determination of the likely site of origin of SCC, although this is feasible for adenocarcinomas in the majority of cases (19). Some related and heuristic studies have been performed in recent years. For example, Huang et al performed immunohistochemical staining to compare the expression profile of the epithelial-mesenchymal transition markers between a metastatic lesion of the duodenum and primary SCC of the hypopharynx (20). Therefore, the differentiation between primary and metastatic nasal vestibular SCC currently depends mainly on clinical and histological examination. The clinical and histological evidence confirmed that the second tumor of the nasal vestibule was primary in this case. Based on these results, the patient was diagnosed with double primary cancer of the lung and nasal vestibule.

The incidence of second cancers has increased (21-23). A study on data from the European Cancer Registry reported an overall incidence of double primary cancer of $6.3 \%$ (range, $0.4-12.9 \%$ ) (24); Utada et al reported that the proportion of patients with a second primary tumor during the follow-up period was $8.1 \%$ (median, 1.8 years; mean, 4.3 years) (25). Filali et al reported variable proportions of double primary cancer, ranging between 6.1 and $10.5 \%$ (26). Furthermore, the risk of second primary cancer among cancer patient was higher compared with the risk of cancer in the general population $(21-22,27-28)$. For example, among second primary cancers, $19.0 \%$ were diagnosed within 3 months after the diagnosis of the first primary cancer (25). Another study reported that cancer patients in Connecticut had a $31 \%$ higher risk of developing a subsequent cancer (28). The increased incidence is not only due to the population aging, but it may also be attributed to the advances in medical technology, particularly diagnostic techniques and cancer treatment modalities (29). The mechanism underlying the development of multiple primary cancers has not been fully elucidated; it may be related to the late effects of treatment for the first primary cancer, or risk factors common to the first and subsequent primary cancer, such as environmental and lifestyle factors, prolonged exposure to carcinogens, radiotherapy and chemotherapy, 

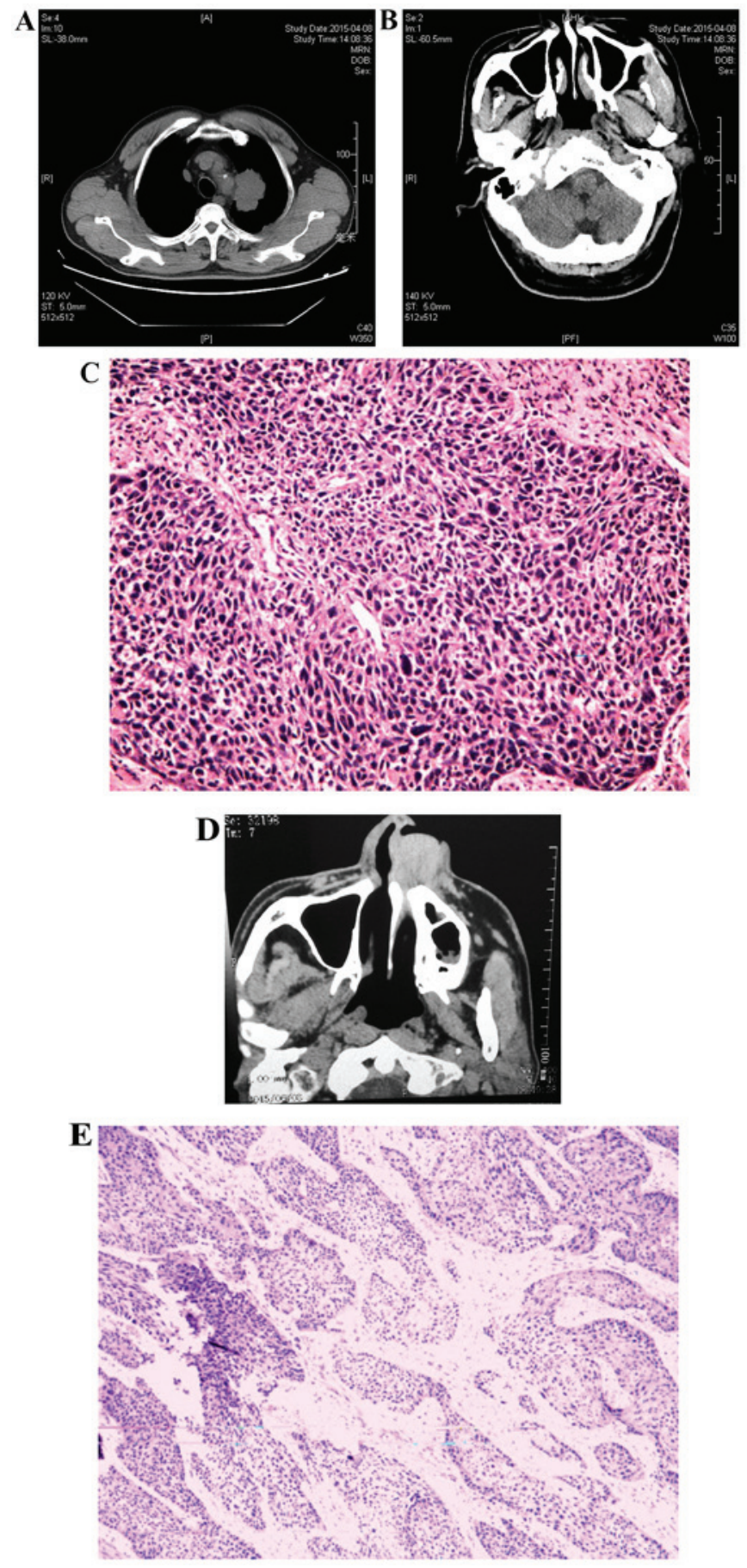

Figure 1. (A) Computed tomography (CT) scan showing a 5.3x3.7-cm mass of the left upper pulmonary lobe; (B) CT scan showing no obvious brain lesions prior to surgery; (C) the left upper pulmonary lobectomy and histopathological examination revealed a poorly differentiated squamous cell carcinoma (pT3N0M0, IIb); (D) CT scan showing a large mass of the left nasal vestibule; (E) Tru-Cut biopsies from the nasal lesion and histopathological examination revealed a moderately differentiated squamous cell carcinoma (T2).

cellular and humoral immunity deficit and individual genetic susceptibility (30-33).

Our patient underwent major invasive surgery for lung cancer. In addition, he received one cycle of adjuvant chemotherapy, which weakened the body's immune system, increasing the risk of cancer. Other important factors were the 20 -year history of heavy smoking and alcohol consumption. A study reported that the major risk factor shared between first and second primary cancers is smoking; a synergetic effect between smoking and drinking may also contribute to the development of multiple primary cancers (34-36). However, a definitive causative factor of double primary cancer has not been clearly determined; further detailed investigation on protective and risk factors is required.

For SCC of the lung, platinum-based regimens are considered as the mainstay of treatment (37). However, in our patient, although nedaplatin and gemcitabine are effective in the treatment of SCC of lung cancer, they confer no therapeutic benefit for SCC of the nasal vestibule. Wray et al (9) evaluated the University of Florida treatment of nasal vestibular carcinoma over a period of $>40$ years and reported excellent disease control with radiotherapy alone in patients with T1-T2 and favorable T3-T4. Radiotherapy and surgery result in improved likelihood of cure for patients with advanced T4 lesions. Vital et al (38) reported that radiotherapy is a valuable alternative treatment option avoiding potential cosmetic disadvantages. Thus, we recommend treatment with radiotherapy rather than chemotherapy for patients with SCC of the nasal vestibule. Planning to receive treatment at a local hospital, the patient was discharged. After 2 months of follow-up, the volume of the nose tumor was significantly reduced through radiotherapy and immunity-enhancing treatment.

A study in Japan reported that the esophagus, ovary, larynx, pharynx and mouth were the most frequent sites for developing a second primary cancer (21). In Queensland, Australia, from 1997 to 2001, second primary cancers of the head and neck were frequent, whereas those of the esophagus and ovary were not as common (22). However, the most common sites for occurrence second primary cancer may not be as important; all clinicians should bear in mind that, when a cancer patient is experiencing discomfort in these sites, the possibility of double or multiple primary cancers must be considered and the frequency of medical examinations and complementary tests must be increased, with the aim to improve the diagnostic rate of such patients and avoid misdiagnosis and treatment delay. Although multiple primary cancers have rarely been reported in China, their incidence is considered to be on the increase. Early detection is crucial to provide optimal treatment and improve the survival rate of cancer patients.

In conclusion, cancer patients are at a higher risk of a second primary cancers than the general population. The esophagus, ovary, and head and neck were the most frequent sites for developing a second primary cancer. Therefore, cancer patients should be under close scrutiny, particularly shortly after the diagnosis of their first primary cancer. Early detection of a silent tumor is meaningful in selecting the optimal treatment option available. Platinum-based chemotherapy is the mainstay of treatment in SCC of the lung; however, for SCC of the head and neck, treatment with radiotherapy is recommended.

Written informed consent was obtained from the patient for publication of this case report and any accompanying images.

\section{References}

1. Moertel CG, Dockerty MB and Baggenstoss AH: Multiple primary malignant neoplasms. II. Tumors of different tissues or organs. Cancer 14: 231-237, 1961. 
2. Billroth T: General Surgical Pathology and Therapeutics in 51 Vorlesungen: A textbook for students and physicians in fifty-one lectures. 14th edition. Berlin, DE: G. Rerimer, 1889.

3. Warren S and Gates O: Multiple primary malignant tumors: Surgery of literature and statistical study. Am J Cancer 16 $1358-1414,1932$.

4. Tsukuma H, Fujimoto I, Hanai A, Hiyama T, Kitagawa T and Kinoshita N: Incidence of second primary cancers in Osaka residents, Japan, with special reference to cumulative and relative risks. Jpn J Cancer Res 85: 339-345, 1994.

5. Jemal A, Bray F, Center M, Ferlay J, Ward E and Forman D: Global cancer statistics. CA Cancer J Clin 61: 69-90, 2011.

6. Rossi A, Chiodini P, Sun JM, O'Brien ME, von Plessen C, Barata F, Park K, Popat S, Bergman B, Parente B, et al: Six versus fewer planned cycles of first-line platinum-based chemotherapy for non-small-cell lung cancer: A systematic review and meta-analysis of individual patient data. Lancet Oncol 15: 1254-1262, 2014.

7. Thanendrarajan S, Nowak M, Abken H and Schmidt-Wolf IG: Combining cytokine-induced killer cells with vaccination in cancer immunotherapy: More than one plus one. Leuk Res 35: 1136-1142, 2011

8. Han RX, Liu X, Pan P, Jia YJ and Yu JC: Effectiveness and safety of chemotherapy combined with dendritic cells co-cultured with cytokine-induced killer cells in the treatment of advanced non-small-cell lung cancer: A systematic review and meta-analysis. PLoS One 9: e108958, 2014.

9. Wray J, Morris CG, Kirwan JM, Amdur RJ, Werning JW, Dziegielewski PT and Mendenhall WM: Radiation therapy for nasal vestibule squamous cell carcinoma: A 40-year experience. Eur Arch Otorhinolaryngol 2015 Mar 13 (Epub ahead of print).

10. Jeannon JP, Riddle PJ, Irish J, O'Sullivan B, Brown DH and Gullane P: Prognostic indicators in carcinoma of the nasal vestibule. Clin Otolaryngol 32: 19-23, 2007.

11. Kummer E, Rasch CR, Keus RB, Tan IB and Balm AJ: T stage as prognostic factor in irradiated localized squamous cell carcinoma of the nasal vestibule. Head Neck 24: 268-273, 2002.

12. Agger A, von Buchwald C, Madsen AR, Yde J, Lesnikova I, Christensen CB, Foghsgaard S, Christensen TB, Hansen HS Larsen S, et al: Squamous cell carcinoma of the nasal vestibule 1993-2002: A nationwide retrospective study from DAHANCA. Head Neck 31: 1593-1599, 2009.

13. Wong CS, Cummings BJ, Elhakim T and Briant TD: External irradiation for squamous cell carcinoma of the nasal vestibule. Int J Radiat Oncol Biol Phys 12: 1943-1946, 1986.

14. Patel P, Tiwari R, Karim AB, Nauta JJ and Snow GB: Squamous cell carcinoma of the nasal vestibule. J Laryngol Otol 106: 332-336, 1992

15. Fornelli RA, Fedok FG, Wilson EP and Rodman SM: Squamous cell carcinoma of the anterior nasal cavity: A dual institution review. Otolaryngol Head Neck Surg 123: 207-210, 2000.

16. Samaha M, Yoskovitch A, Hier MP and Black MJ: Squamous cell carcinoma of the nasal vestibule. J Otolaryngol 29: 98-101, 2000.

17. Dowley A, Hoskison E, Allibone R and Jones NS: Squamous cell carcinoma of the nasal vestibule: A 20-year case series and literature review. J Laryngol Otol 122: 1019-1023, 2008.

18. Quinn FB Jr and Mccabe BF: Laryngeal metastases from malignant tumors in distant organs. Ann Otol Rhinol Laryngol 66: 139-143, 1957.

19. Oien KA: Pathologic evaluation of unknown primary cancer. Semin Oncol 36: 8-37, 2009.

20. Huang YC, Chang PM, Lee IC, Yang CF, Tzeng $\mathrm{CH}$ and Yang MH: Duodenal squamous cell carcinoma derived from the hypopharynx: Immunohistochemical assessment of metastatic mechanisms. Gastrointest Endosc 72: 460-463, 2010.
21. Tabuchi T, Ito Y, Ioka A, Miyashiro I and Tsukuma H: Incidence of metachronous second primary cancers in Osaka, Japan: Update of analyses using population-based cancer registry data. Cancer Sci 103: 1111-1120, 2012.

22. Youlden DR and Baade PD: The relative risk of second primary cancers in Queensland, Australia: A retrospective cohort study. BMC Cancer 11: 83, 2011.

23. Freeman HJ: Triple metachronous colon cancer. World J Gastroenterol 19: 4443-4444, 2013.

24. Rosso S, De Angelis R, Ciccolallo L, Carrani E, Soerjomataram I, Grande E, Zigon $\mathrm{G}$ and Brenner H; EUROCARE Working Group: Multiple tumours in survival estimates. Eur J Cancer 45: 1080-1094, 2009.

25. Utada M, Ohno Y, Hori M and Soda M: Incidence of multiple primary cancers and interval between first and second primary cancers. Cancer Sci 105: 890-896, 2014.

26. Filali K, Hédelin G, Schaffer P, Estève J, Arveux P, Bouchardy C, Exbrayat C, Faivre J, Lévi F, Macé-Lesech J, et al: Multiple primary cancers and estimation of the incidence rates and trends. Eur J Cancer 32A: 683-690, 1996.

27. Crocetti E, Buiatti E and Falini P; Italian Multiple Primary Cancer Working Group: Multiple primary cancer incidence in Italy. Eur J Cancer 37: 2449-2456, 2001.

28. Curtis RE, Boice JD Jr, Kleinerman RA, Flannery JT and Fraumeni JF Jr: Summary: Multiple primary cancers in Connecticut 1935-82. Natl Cancer Inst Monogr 68: 219-242, 1985.

29. Ikeda Y, Tsukuda M, Ishitoya J, Arai Y, Matsuda H, Katori H, Taguchi T, Yoshida T, Ono M, Sakuma Y, et al: Four cases of simultaneous triple primary cancers of the hypopharynx, esophagus and stomach. Otolaryngol Head Neck Surg 132: 788-793, 2005

30. Travis LB, Rabkin CS, Brown LM, Allan JM, Alter BP, Ambrosone CB, Begg CB, Caporaso N, Chanock S, DeMichele A, et al: Cancer survivorship - genetic susceptibility and second primary cancers: Research strategies and recommendations. J Natl Cancer Inst 98: 15-25, 2006.

31. Travis LB: The epidemiology of second primary cancers. Cancer Epidemiol Biomarkers Prev 15: 2020-2067, 2006.

32. Kuligina E, Reiner A, Imyanitov EN and Begg CB: Evaluating cancer epidemiologic risk factors using multiple primary malignancies. Epidemiology 21: 366-372, 2010.

33. Jiao F, Hu H and Wang LW: Quadruple primary malignancy patient with survival time more than 20 years. World J Gastroenterol 19: 1498-1501, 2013.

34. Adami HO, Hunter D and Trichopoulos D (eds): Textbook of Cancer Epidemiology. 2nd edition. Oxford University Press, New York, 2002

35. Hiyama T, Sato T, Yoshino K, Tsukuma H, Hanai A and Fujimoto I: Second primary cancer following laryngeal cancer with special reference to smoking habits. Jpn J Cancer Res 83: 334-339, 1992.

36. Botteri E, Iodice S, Bagnardi V, Raimondi S, Lowenfels AB and Maisonneuve P: Smoking and colorectal cancer: A meta-analysis. JAMA 300: 2765-2778, 2008.

37. DeVita VT Jr and Rosenberg SA: Two hundred years of cancer research. N Engl J Med 366: 2207-2214, 2012.

38. Vital D, Morand G, Huber GF, Studer G and Holzmann D: Outcome in squamous cell carcinoma of the nasal vestibule: A single center experience. Head Neck 37: 46-51, 2015. 